\title{
Promotion and Evacuation
}

\author{
Richard P. Stanley* \\ Department of Mathematics \\ M.I.T., Cambridge, Massachusetts, USA \\ rstan@math.mit.edu \\ Submitted: Jul 22, 2008; Accepted: Apr 21, 2009; Published: Apr 27, 2009 \\ Mathematics Subject Classifications: 06A07 \\ Dedicated to Anders Björner on the occasion of his sixtieth birthday.
}

\begin{abstract}
Promotion and evacuation are bijections on the set of linear extensions of a finite poset first defined by Schützenberger. This paper surveys the basic properties of these two operations and discusses some generalizations. Linear extensions of a finite poset $P$ may be regarded as maximal chains in the lattice $J(P)$ of order ideals of $P$. The generalizations concern permutations of the maximal chains of a wider class of posets, or more generally bijective linear transformations on the vector space with basis consisting of the maximal chains of any poset. When the poset is the lattice of subspaces of $\mathbb{F}_{q}^{n}$, then the results can be stated in terms of the expansion of certain Hecke algebra products.
\end{abstract}

\section{Introduction.}

Promotion and evacuation are bijections on the set of linear extensions of a finite poset. Evacuation first arose in the theory of the RSK algorithm, which associates a permutation in the symmetric group $\mathfrak{S}_{n}$ with a pair of standard Young tableaux of the same shape [31, pp. 320-321]. Evacuation was described by M.-P. Schützenberger [25] in a direct way not involving the RSK algorithm. In two follow-up papers [26][27] Schützenberger extended the definition of evacuation to linear extensions of any finite poset. Evacuation is described in terms of a simpler operation called promotion. Schützenberger established many fundamental properties of promotion and evacuation, including the result that evacuation is an involution. Schützenberger's work was simplified by Haiman [15] and

*This material is based upon work supported by the National Science Foundation under Grant No. 0604423. Any opinions, findings and conclusions or recommendations expressed in this material are those of the author and do not necessarily reflect those of the National Science Foundation. 
Malvenuto and Reutenauer [19], and further work on evacuation was undertaken by a number of researchers (discussed in more detail below).

In this paper we will survey the basic properties of promotion and evacuation. We will then discuss some generalizations. In particular, the linear extensions of a finite poset $P$ correspond to the maximal chains of the distributive lattice $J(P)$ of order ideals of $P$. We will extend promotion and evacuation to bijections on the vector space whose basis consists of all maximal chains of a finite graded poset $Q$. The case $Q=B_{n}(q)$, the lattice of subspaces of the vector space $\mathbb{F}_{q}^{n}$, leads to some results on expanding a certain product in the Hecke algebra $\mathcal{H}_{n}(q)$ of $\mathfrak{S}_{n}$ in terms of the standard basis $\left\{T_{w}: w \in \mathfrak{S}_{n}\right\}$.

I am grateful to Kyle Petersen and two anonymous referees for many helpful comments on earlier versions of this paper.

\section{Basic results.}

We begin with the original definitions of promotion and evacuation due to Schützenberger. Let $P$ be a $p$-element poset. We write $s \lessdot t$ if $t$ covers $s$ in $P$, i.e., $s<t$ and no $u \in P$ satisfies $s<u<t$. The set of all linear extensions of $P$ is denoted $\mathcal{L}(P)$. Schützenberger regards a linear extension as a bijection $f: P \rightarrow[p]=\{1,2, \ldots, p\}$ such that if $s<t$ in $P$, then $f(s)<f(t)$. (Actually, Schützenberger considers bijections $f: P \rightarrow\{k+1, k+2, \ldots, k+p\}$ for some $k \in \mathbb{Z}$, but we slightly modify his approach by always ensuring that $k=0$.) Think of the element $t \in P$ as being labelled by $f(t)$. We now define a bijection $\partial: \mathcal{L}(P) \rightarrow \mathcal{L}(P)$, called promotion, as follows. Let $t_{1} \in P$ satisfy $f\left(t_{1}\right)=1$. Remove the label 1 from $t_{1}$. Among the elements of $P$ covering $t_{1}$, let $t_{2}$ be the one with the smallest label $f\left(t_{2}\right)$. Remove this label from $t_{2}$ and place it at $t_{1}$. (Think of "sliding" the label $f\left(t_{2}\right)$ down from $t_{2}$ to $t_{1}$.) Now among the elements of $P$ covering $t_{2}$, let $t_{3}$ be the one with the smallest label $f\left(t_{3}\right)$. Slide this label from $t_{3}$ to $t_{2}$. Continue this process until eventually reaching a maximal element $t_{k}$ of $P$. After we slide $f\left(t_{k}\right)$ to $t_{k-1}$, label $t_{k}$ with $p+1$. Now subtract 1 from every label. We obtain a new linear extension $f \partial \in \mathcal{L}(P)$. Note that we let $\partial$ operate on the right. Note also that $t_{1} \lessdot t_{2} \lessdot \cdots \lessdot t_{k}$ is a maximal chain of $P$, called the promotion chain of $f$. Figure 1 (a) shows a poset $P$ and a linear extension $f$. The promotion chain is indicated by circled dots and arrows. Figure 1(b) shows the labeling after the sliding operations and the labeling of the last element of the promotion chain by $p+1=10$. Figure $1(\mathrm{c})$ shows the linear extension $f \partial$ obtained by subtracting 1 from the labels in Figure 1(b).

It should be obvious that $\partial: \mathcal{L}(P) \rightarrow \mathcal{L}(P)$ is a bijection. In fact, let $\partial^{*}$ denote dual promotion, i.e., we remove the largest label $p$ from some element $u_{1} \in P$, then slide the largest label of an element covered by $u_{1}$ up to $u_{1}$, etc. After reaching a minimal element $u_{k}$, we label it by 0 and then add 1 to each label, obtaining $f \partial^{*}$. It is easy to check that

$$
\partial^{-1}=\partial^{*}
$$

We next define a variant of promotion called evacuation. The evacuation of a linear extension $f \in \mathcal{L}(P)$ is denoted $f \epsilon$ and is another linear extension of $P$. First compute $f \partial$. 


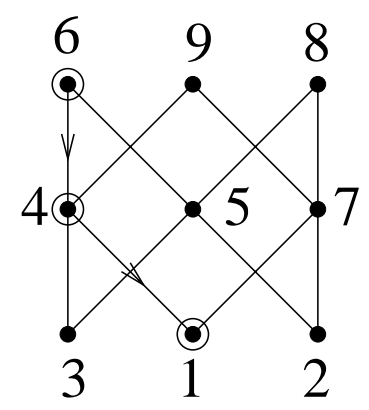

(a)

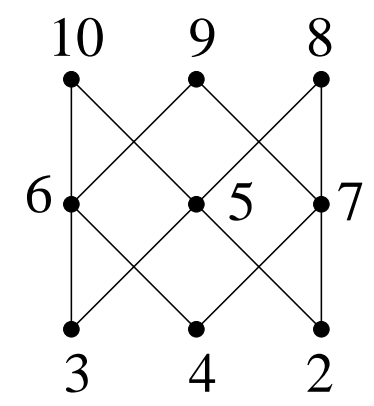

(b)

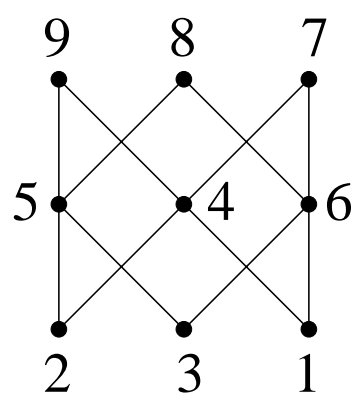

(c)

Figure 1: The promotion operator $\partial$ applied to a linear extension
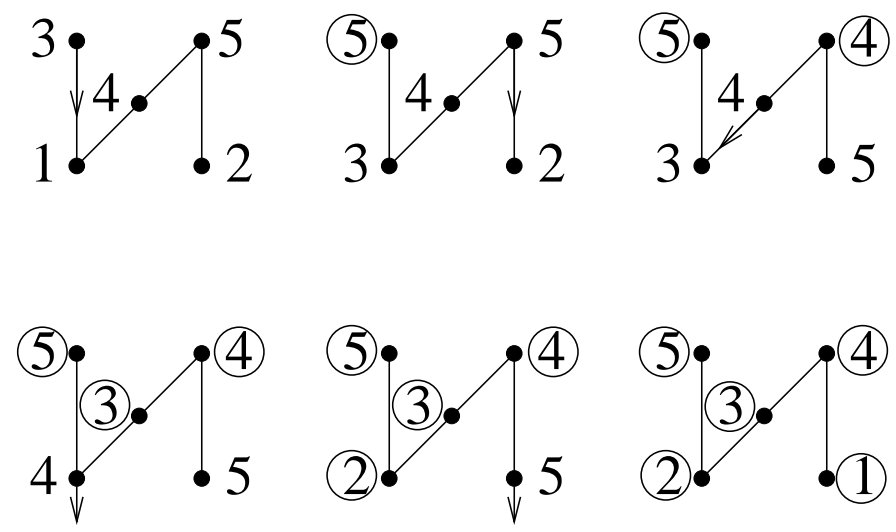

Figure 2: The evacuation of a linear extension $f$

Then "freeze" the label $p$ into place and apply $\partial$ to what remains. In other words, let $P_{1}$ consist of those elements of $P$ labelled $1,2, \ldots, p-1$ by $f \partial$, and apply $\partial$ to the restriction of $\partial f$ to $P_{1}$. Then freeze the label $p-1$ and apply $\partial$ to the $p-2$ elements that remain. Continue in this way until every element has been frozen. Let $f \epsilon$ be the linear extension, called the evacuation of $f$, defined by the frozen labels.

Note. A standard Young tableau of shape $\lambda$ can be identified in an obvious way with a linear extension of a certain poset $P_{\lambda}$. Evacuation of standard Young tableaux has a nice geometric interpretation connected with the nilpotent flag variety. See van Leeuwen [18, §3] and Tesler [36, Thm. 5.14].

Figure 2 illustrates the evacuation of a linear extension $f$. The promotion paths are shown by arrows, and the frozen elements are circled. For ease of understanding we don't subtract 1 from the unfrozen labels since they all eventually disappear. The labels are always frozen in descending order $p, p-1, \ldots, 1$. Figure 3 shows the evacuation of $f \epsilon$, where $f$ is the linear extension of Figure 2. Note that (seemingly) miraculously we have $f \epsilon^{2}=f$. This example illustrates a fundamental property of evacuation given by Theorem 2.1(a) below.

We can define dual evacuation analogously to dual promotion. In symbols, if $f \in \mathcal{L}(P)$ 

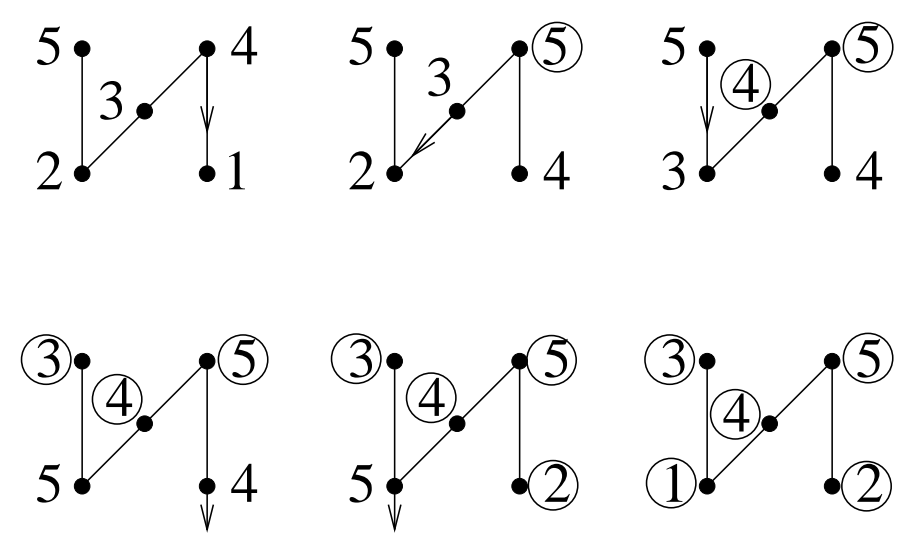

Figure 3: The linear extension $\operatorname{evac}(\operatorname{evac}(f))$

then define $f^{*} \in \mathcal{L}\left(P^{*}\right)$ by $f^{*}(t)=p+1-f(t)$. Thus

$$
f \epsilon^{*}=\left(f^{*} \epsilon\right)^{*}
$$

We can now state three of the four main results obtained by Schützenberger.

Theorem 2.1. Let $P$ be a p-element poset. Then the operators $\epsilon, \epsilon^{*}$, and $\partial$ satisfy the following properties.

(a) Evacuation is an involution, i.e., $\epsilon^{2}=1$ (the identity operator).

(b) $\partial^{p}=\epsilon \epsilon^{*}$

(c) $\partial \epsilon=\epsilon \partial^{-1}$

Theorem 2.1 can be interpreted algebraically as follows. The bijections $\epsilon$ and $\epsilon^{*}$ generate a subgroup $D_{P}$ of the symmetric group $\mathfrak{S}_{\mathcal{L}(P)}$ on all the linear extensions of $P$. Since $\epsilon$ and (by duality) $\epsilon^{*}$ are involutions, the group they generate is a dihedral group $D_{P}$ (possibly degenerate, i.e., isomorphic to $\{1\}, \mathbb{Z} / 2 \mathbb{Z}$, or $\mathbb{Z} / 2 \mathbb{Z} \times \mathbb{Z} / 2 \mathbb{Z}$ ) of order 1 or $2 m$ for some $m \geq 1$. If $\epsilon$ and $\epsilon^{*}$ are not both trivial (which can only happen when $P$ is a chain), so they generate a group of order $2 m$, then $m$ is the order of $\partial^{p}$. In general the value of $m$, or more generally the cycle structure of $\partial^{p}$, is mysterious. For a few cases in which more can be said, see Section 4.

The main idea of Haiman [15, Lemma 2.7, and page 91] (further developed by Malvenuto and Reutenauer [19]) for proving Theorem 2.1 is to write linear extensions as words rather than functions and then to describe the actions of $\partial$ and $\epsilon$ on these words. The proof then becomes a routine algebraic computation. Let us first develop the necessary algebra in a more general context.

Let $G$ be the group with generators $\tau_{1}, \ldots, \tau_{p-1}$ and relations

$$
\begin{aligned}
\tau_{i}^{2}=1, & 1 \leq i \leq p-1 \\
\tau_{i} \tau_{j}=\tau_{j} \tau_{i}, & \text { if }|i-j|>1
\end{aligned}
$$


Some readers will recognize that $G$ is an infinite Coxeter group ( $p \geq 3$ ) with the symmetric group $\mathfrak{S}_{p}$ as a quotient. Define the following elements of $G$ :

$$
\begin{aligned}
\delta & =\tau_{1} \tau_{2} \cdots \tau_{p-1} \\
\gamma=\gamma_{p} & =\tau_{1} \tau_{2} \cdots \tau_{p-1} \cdot \tau_{1} \tau_{2} \cdots \tau_{p-2} \cdots \tau_{1} \tau_{2} \cdot \tau_{1} \\
\gamma^{*} & =\tau_{p-1} \tau_{p-2} \cdots \tau_{1} \cdot \tau_{p-1} \tau_{p-2} \cdots \tau_{2} \cdots \tau_{p-1} \tau_{p-2} \cdot \tau_{p-1}
\end{aligned}
$$

Lemma 2.2. In the group $G$ we have the following identities:

(a) $\gamma^{2}=\left(\gamma^{*}\right)^{2}=1$

(b) $\delta^{p}=\gamma \gamma^{*}$

(c) $\delta \gamma=\gamma \delta^{-1}$.

Proof. (a) Induction on $p$. For $p=2$, we need to show that $\tau_{1}^{2}=1$, which is given. Now assume for $p-1$. Then

$$
\begin{array}{r}
\gamma_{p}^{2}=\tau_{1} \tau_{2} \cdots \tau_{p-1} \cdot \tau_{1} \cdots \tau_{p-2} \cdots \tau_{1} \tau_{2} \tau_{3} \cdot \tau_{1} \tau_{2} \cdot \tau_{1} \\
\cdot \tau_{1} \tau_{2} \cdots \tau_{p-1} \cdot \tau_{1} \cdots \tau_{p-2} \cdots \tau_{1} \tau_{2} \tau_{3} \cdot \tau_{1} \tau_{2} \cdot \tau_{1} .
\end{array}
$$

We can cancel the two middle $\tau_{1}$ 's since they appear consecutively. We can then cancel the two middle $\tau_{2}$ 's since they are now consecutive. We can then move one of the middle $\tau_{3}$ 's past a $\tau_{1}$ so that the two middle $\tau_{3}$ 's are consecutive and can be cancelled. Now the two middle $\tau_{4}$ 's can be moved to be consecutive and then cancelled. Continuing in this way, we can cancel the two middle $\tau_{i}$ 's for all $1 \leq i \leq p-1$. When this cancellation is done, what remains is the element $\gamma_{p-1}^{2}$, which is 1 by induction.

(b,c) Analogous to (a). Details are omitted.

Proof of Theorem 2.1. A glance at Theorem 2.1 and Lemma 2.2 makes it obvious that they should be connected. To see this connection, regard the linear extension $f \in \mathcal{L}(P)$ as the word (or permutation of $P$ ) $f^{-1}(1), \ldots, f^{-1}(p)$. For $1 \leq i \leq p-1$ define operators $\tau_{i}: \mathcal{L}(P) \rightarrow \mathcal{L}(P)$ by

$$
\tau_{i}\left(u_{1} u_{2} \cdots u_{p}\right)=\left\{\begin{aligned}
& u_{1} u_{2} \cdots u_{p}, \text { if } u_{i} \text { and } u_{i+1} \text { are } \\
& \text { comparable in } P \\
& u_{1} u_{2} \cdots u_{i+1} u_{i} \cdots u_{p}, \text { otherwise }
\end{aligned}\right.
$$

Clearly $\tau_{i}$ is a bijection, and the $\tau_{i}$ 's satisfy the relations (1). By Lemma 2.2, the proof of Theorem 2.1 follows from showing that

$$
\partial=\delta:=\tau_{1} \tau_{2} \cdots \tau_{p-1}
$$

Note that if $f=u_{1} u_{2} \cdots u_{p}$, then $f \delta$ is obtained as follows. Let $j$ be the least integer such that $j>1$ and $u_{1}<u_{j}$. Since $f$ is a linear extension, the elements $u_{2}, u_{3}, \ldots, u_{j-1}$ are incomparable with $u_{1}$. Move $u_{1}$ so it is between $u_{j-1}$ and $u_{j}$. (Equivalently, cyclically shift the sequence $u_{1} u_{2} \cdots u_{j-1}$ one unit to the left.) Now let $k$ be the least integer such 


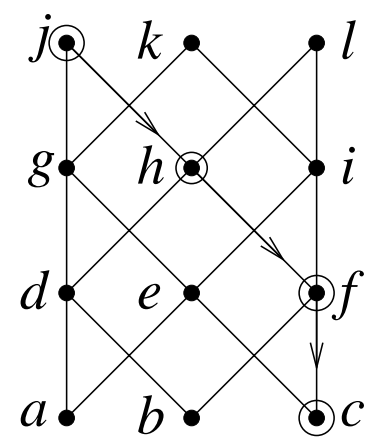

Figure 4: The promotion chain of the linear extension cabdfeghilk

that $k>j$ and $u_{j}<u_{k}$. Move $u_{j}$ so it is between $u_{k-1}$ and $u_{k}$. Continue in this way reaching the end. For example, let $z$ be the linear extension cabdfeghjilk of the poset in Figure 4 (which also shows the promotion chain for this linear extension). We factor $z$ from left-to-right into the longest factors for which the first element of each factor is incomparable with the other elements of the factor:

$$
z=\mathrm{cabd} \cdot \mathrm{feg} \cdot h \cdot j i l k .
$$

Cyclically shift each factor one unit to the left to obtain $z \delta$ :

$$
z \delta=a b d c \cdot e g f \cdot h \cdot i l k j=a b d c e g f h k i l j .
$$

Now consider the process of promoting the linear extension $f$ of the previous paragraph, given as a function by $f\left(u_{i}\right)=i$ and as a word by $u_{1} u_{2} \cdots u_{p}$. The elements $u_{2}, \ldots, u_{j-1}$ are incomparable with $u_{1}$ and thus will have their labels reduced by 1 after promotion. The label $j$ of $u_{j}$ (the least element in the linear extension $f$ greater than $u_{1}$ ) will slide down to $u_{1}$ and be reduced to $j-1$. Hence $f \partial=u_{2} u_{3} \cdots u_{j-1} u_{1} \cdots$. Exactly analogous reasoning applies to the next step of the promotion process, when we slide the label $k$ of $u_{k}$ down to $u_{j}$. Continuing in this manner shows that $z \delta=z \partial$, completing the proof of Theorem 2.1.

Note. The operators $\tau_{i}: \mathcal{L}(P) \rightarrow \mathcal{L}(P)$ have the additional property that $\left(\tau_{i} \tau_{i+1}\right)^{6}=$ 1 , but we see no way to exploit this fact.

Theorem 2.1 states three of the four main results of Schützenberger. We now discuss the fourth result. Let $f: P \rightarrow[p]$ be a linear extension, and apply $\partial p$ times, using Schützenberger's original description of $\partial$ given at the beginning of this section. Say $f\left(t_{1}\right)=p$. After applying sufficiently many $\partial$ 's, the label of $t_{1}$ will slide down to a new element $t_{2}$ and then be decreased by 1 . Continuing to apply $\partial$, the label of $t_{2}$ will eventually slide down to $t_{3}$, etc. Eventually we will reach a minimal element $t_{j}$ of $P$. We call the chain $\left\{t_{1}, t_{2}, \ldots, t_{j}\right\}$ the principal chain of $f$ (equivalent to Schützenberger's definition of "orbit"), denoted $\rho(f)$. For instance, let $f$ be the linear extension of Figure 5(b) of the poset of Figure 5(a). After applying $\partial$, the label 5 of $e$ slides down to $d$ and becomes 4 . Two more applications of $\partial$ cause the label 3 to $d$ to slide down to $a$. Thus $\rho(f)=\{a, d, e\}$. 


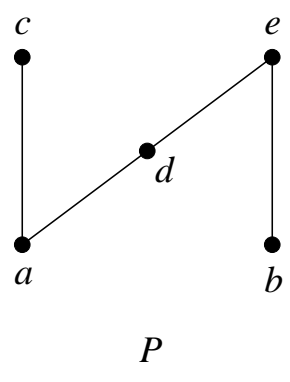

(a)

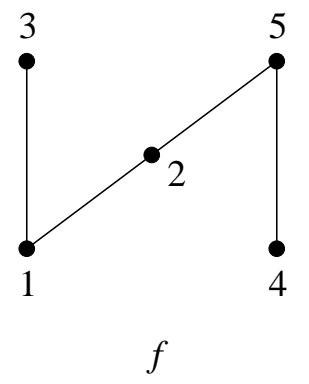

(b)

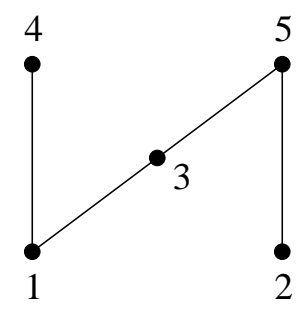

$f \varepsilon$

(c)

Figure 5: A poset $P$ with a linear extension and its evacuation

Now apply $\partial$ to the evacuation $f \epsilon$. Let $\sigma(f \epsilon)$ be the chain of elements of $P$ along which labels slide, called the trajectory of $f$. For instance, Figure $5(\mathrm{c})$ shows $f \epsilon$, where $f$ is given by Figure 5(b). When we apply $\partial$ to $f \epsilon$, the label 1 of $a$ is removed, the label 3 of $d$ slides to $a$, and the label 5 of $e$ slides to $d$. Schützenberger's fourth result is the following.

Theorem 2.3. For any finite poset $P$ and $f \in \mathcal{L}(P)$ we have $\rho(f)=\sigma(f \epsilon)$.

Proof (sketch). Regard the linear extension $f \partial^{i}$ of $P$ as the word $u_{i 1} u_{i 2} \cdots u_{i p}$. It is clear that

$$
\rho(f)=\left\{u_{0 p}, u_{1, p-1}, u_{2, p-2}, \ldots, u_{p-1,1}\right\}
$$

(where multiple elements are counted only once). On the other hand, let $\psi_{j}=\tau_{1} \tau_{2} \cdots \tau_{p-j}$, and regard the linear extension $f \psi_{1} \psi_{2} \cdots \psi_{i}$ as the word $v_{i 1} v_{i 2} \cdots v_{i p}$. It is clear that $v_{i j}=u_{i j}$ if $i+j \leq p$. In particular, $u_{i, p-i}=v_{i, p-i}$. Moreover, $f \epsilon=v_{2, p}, v_{3, p-1}, \ldots, v_{p+1,1}$. We leave to the reader to check that the elements of $\rho(f)$ written in increasing order, say $z_{1}<z_{2}<\cdots<z_{k}$, form a subsequence of $f \epsilon$, since $u_{i, p-i}=v_{i, p-i}$. Moreover, the elements of $f \epsilon$ between $z_{j}$ and $z_{j+1}$ are incomparable with $z_{j}$. Hence when we apply $\partial$ to $f \epsilon$, the element $z_{1}$ moves to the right until reaching $z_{2}$, then $z_{2}$ moves to the right until reaching $z_{3}$, etc. This is just what it means for $\sigma(f \epsilon)=\left\{z_{1}, \ldots, z_{k}\right\}$, completing the proof.

Promotion and evacuation can be applied to other properties of linear extensions. We mention three such results here. For the first, let $e(P)$ denote the number of linear extensions of the finite poset $P$. If $A$ is the set of minimal (or maximal) elements of $P$, then it is obvious that

$$
e(P)=\sum_{t \in A} e(P-t)
$$

An antichain of $P$ is a set of pairwise incomparable elements of $P$. Edelman, Hibi, and Stanley [9] use promotion to obtain the following generalization of equation (3) (a special case of an even more general theorem).

Theorem 2.4. Let $A$ be an antichain of $P$ that intersects every maximal chain. Then

$$
e(P)=\sum_{t \in A} e(P-t)
$$


The second application of promotion and evacuation is to the theory of sign balance. Fix an ordering $t_{1}, \ldots, t_{p}$ of the elements of $P$, and regard a linear extension of $f: P \rightarrow[p]$ as the permutation $w$ of $P$ given by $w\left(t_{i}\right)=f^{-1}(i)$. A finite poset $P$ is sign balanced if it has the same number of even linear extensions as odd linear extensions. It is easy to see that the property of being sign balanced does not depend on the ordering $t_{1}, \ldots, t_{p}$. While it is difficult in general to understand the cycle structure of the operator $\partial$ (regarded as a permutation of the set of all linear extensions $f$ of $P$ ), there are situations when we can analyze its effect on the parity of $f$. Moreover, Theorem 3.1 determines the cycle structure of $\epsilon$. This idea leads to the following result of Stanley [32, Cor. 2.2 and 2.4].

Theorem 2.5. (a) Let $\# P=p$, and suppose that the length $\ell$ of every maximal chain of $P$ satisfies $p \equiv \ell(\bmod 2)$. Then $P$ is sign-balanced.

(b) Suppose that for all $t \in P$, the lengths of all maximal chains of the principal order ideal $\Lambda_{t}:=\{s \in P: s \leq t\}$ have the same parity. Let $\nu(t)$ denote the length of the longest chain of $\Lambda_{t}$, and set $\Gamma(P)=\sum_{t \in P} \nu(t)$. If $\left(\begin{array}{l}p \\ 2\end{array}\right) \equiv \Gamma(P)(\bmod 2)$ then $P$ is sign-balanced.

Our final application is related to an operation $\psi$ on antichains $A$ of a finite poset $P$. Let

$$
I_{A}=\{s \in P: s \leq t \text { for some } t \in A\},
$$

the order ideal generated by $A$. Define $A \psi$ to be the set of minimal elements of $P-I_{A}$. The operation $\psi$ is a bijection on the set $\mathcal{A}(P)$ of antichains of $P$, and there is considerable interest in determining the cycle structure of $\psi$ (see, e.g., Cameron [7] and Panyushev [20]). Here we will show a connection with the case $P=\boldsymbol{m} \times \boldsymbol{n}$ (a product of chains of sizes $m$ and $n$ ) and promotion on $\boldsymbol{m}+\boldsymbol{n}$ (where + denotes disjoint union). We first define a bijection $\Phi: \mathcal{L}(\boldsymbol{m}+\boldsymbol{n}) \rightarrow \mathcal{A}(\boldsymbol{m} \times \boldsymbol{n})$. We can write $w \in \mathcal{L}(\boldsymbol{m}+\boldsymbol{n})$ as a sequence $\left(a_{m}, a_{m-1}, \ldots, a_{1}, b_{n}, b_{n-1}, \ldots, b_{1}\right)$ of $m 1$ 's and $n 2$ 's in some order. The position of the 1's indicate when we choose in $w$ (regarded as a word in the elements of $\boldsymbol{m}+\boldsymbol{n}$ ) an element from the first summand $\boldsymbol{m}$. Let $m \geq i_{1}>i_{2}>\cdots>i_{r} \geq 1$ be those indices $i$ for which $a_{i}=2$. Let $j_{1}<j_{2}<\cdots<j_{r}$ be those indices $j$ for which $b_{j}=1$. Regard the elements of $\boldsymbol{m} \times \boldsymbol{n}$ as pairs $(i, j), 1 \leq i \leq m, 1 \leq j \leq n$, ordered coordinatewise. Define

$$
\Phi(w)=\left\{\left(i_{1}, j_{1}\right), \ldots,\left(i_{r}, j_{r}\right)\right\} \in \mathcal{A}(\boldsymbol{m} \times \boldsymbol{n}) .
$$

For instance (writing a bar to show the space between $a_{1}$ and $\left.b_{6}\right), \Phi(1211221 \mid 212211)=$ $\{(6,1),(3,2),(2,5)\}$. It can be checked that $\Phi(w \partial)=\Phi(w) \psi$. Hence $\psi$ on $\boldsymbol{m} \times \boldsymbol{n}$ has the same cycle type as $\partial$ on $\boldsymbol{m}+\boldsymbol{n}$, which is relatively easy to analyze. We omit the details here.

\section{Self-evacuation and $P$-domino tableaux}

In this section we consider self-evacuating linear extensions of a finite poset $P$, i.e., linear extensions $f$ such that $f \epsilon=f$. The main result asserts that the number of self-evacuating $f \in \mathcal{L}(P)$ is equal to two other quantities associated with $P$. We begin by defining these two other quantities. 
An order ideal of $P$ is a subset $I$ such that if $t \in I$ and $s<t$, then $s \in I$. A $P$-domino tableau is a chain $\emptyset=I_{0} \subset I_{1} \subset \cdots \subset I_{r}=P$ of order ideals of $P$ such that $I_{i}-I_{i-1}$ is a two-element chain for $2 \leq i \leq r$, while $I_{1}$ is either a two-element or one-element chain (depending on whether $p$ is even or odd). In particular, $r=\lceil p / 2\rceil$.

Note. In $[32, \S 4]$ domino tableaux were defined so that $I_{r}-I_{r-1}$, rather than $I_{1}$, could have one element. The definition given in the present paper is more consistent with previously defined special cases.

Now assume that the vertex set of $P$ is $[p]$ and that $P$ is a natural partial order, i.e., if $i<j$ in $P$ then $i<j$ in $\mathbb{Z}$. A linear extension of $P$ is thus a permutation $w=a_{1} \cdots a_{p} \in \mathfrak{S}_{p}$. The descent set $D(w)$ of $w$ is defined by

$$
D(w)=\left\{1 \leq i \leq p-1: a_{i}>a_{i+1}\right\},
$$

and the comajor index $\operatorname{comaj}(w)$ is defined by

$$
\operatorname{comaj}(w)=\sum_{i \in D(w)}(p-i) .
$$

(Note. Sometimes the comajor index is defined by $\operatorname{comaj}(w)=\sum_{i \in[p-1]-D(w)} i$, but we will use equation (4) here.) Set

$$
W_{P}^{\prime}(x)=\sum_{w \in \mathcal{L}(P)} x^{\operatorname{comaj}(w)} .
$$

It is known from the theory of $P$-partitions (e.g., $[30, \S 4.5]$ ) that $W_{P}^{\prime}(x)$ depends only on $P$ up to isomorphism.

Note. Usually in the theory of $P$-partitions one works with the major index $\operatorname{maj}(w)=$ $\sum_{i \in D(w)} i$ and with the polynomial $W_{P}(x)=\sum_{w \in \mathcal{L}(P)} x^{\operatorname{maj}(w)}$. Note that if $p$ is even then $\operatorname{comaj}(w) \equiv \operatorname{maj}(w)(\bmod 2)$, so $W_{P}(-1)=W_{P}^{\prime}(-1)$.

Theorem 3.1. Let $P$ be a finite natural partial order. Then the following three quantities are equal.

(i) $W_{P}^{\prime}(-1)$.

(ii) The number of P-domino tableaux.

(iii) The number of self-evacuating linear extensions of $P$.

In order to prove Theorem 3.1, we need one further result about the elements $\tau_{i}$ of equation (1).

Lemma 3.2. Let $G$ be the group of Lemma 2.2. Write

$$
\begin{aligned}
\delta_{i} & =\tau_{1} \tau_{2} \cdots \tau_{i} \\
\delta_{i}^{*} & =\tau_{i} \tau_{i-1} \cdots \tau_{1} .
\end{aligned}
$$

Let $u, v \in G$. The following two conditions are equivalent. 
(i) $u \delta_{1}^{*} \delta_{3}^{*} \cdots \delta_{2 j-1}^{*}=v \delta_{1}^{*} \delta_{3}^{*} \cdots \delta_{2 j-1}^{*} \cdot \delta_{2 j-1} \delta_{2 j-2} \cdots \delta_{2} \delta_{1}$.

(ii) $u \tau_{1} \tau_{3} \cdots \tau_{2 j-1}=v$.

Proof of Lemma 3.2. The proof is a straightforward extension of an argument due to van Leeuwen [17, §2.3] (but not expressed in terms of the group $G$ ) and more explicitly to Berenstein and Kirillov [2]. (About the same time as van Leeuwen, a special case was proved by Stembridge [35] using representation theory. Both Stembridge and BerensteinKirillov deal with semistandard tableaux, while here we consider only the special case of standard tableaux. While standard tableaux have a natural generalization to linear extensions of any finite poset, it is unclear how to generalize semistandard tableaux analogously so that the results of Stembridge and Berenstein-Kirillov continue to hold.) Induction on $j$. The case $j=1$ asserts that $u \tau_{1}=v \tau_{1} \tau_{1}$ if and only if $u \tau_{1}=v$, which is immediate from $\tau_{1}^{2}=1$. Now assume for $j-1$, and suppose that (i) holds. First cancel $\delta_{2 j-1}^{*} \delta_{2 j-1}$ from the right-hand side. Now take the last factor $\tau_{i}$ from each factor $\delta_{i}(1 \leq i \leq 2 j-2)$ on the right-hand side and move it as far to the right as possible. The right-hand side will then end in $\tau_{2 j-2} \tau_{2 j-3} \cdots \tau_{1}=\delta_{2 j-2}^{*}$. The left-hand side ends in $\delta_{2 j-1}^{*}=\tau_{2 j-1} \delta_{2 j-2}^{*}$. Hence we can cancel the suffix $\delta_{2 j-2}^{*}$ from both sides, obtaining

$$
u \delta_{1}^{*} \delta_{3}^{*} \cdots \delta_{2 j-3}^{*} \tau_{2 j-1}=v \delta_{1}^{*} \delta_{3}^{*} \cdots \delta_{2 j-3}^{*} \cdot \delta_{2 j-3} \delta_{2 j-4} \cdots \delta_{2} \delta_{1}
$$

We can now move the rightmost factor $\tau_{2 j-1}$ on the left-hand side of equation (5) directly to the right of $u$. Applying the induction hypothesis with $u$ replaced by $u \tau_{2 j-1}$ yields (ii). The steps are reversible, so (ii) implies (i).

Proof of Theorem 3.1. The equivalence of (i) and (ii) appears (in dual form) in [32, Theorem 5.1(a)]. Namely, let $w=a_{1} \cdots a_{p} \in \mathcal{L}(P)$. Let $i$ be the least nonnegative integer (if it exists) for which

$$
w^{\prime}:=a_{1} \cdots a_{p-2 i-2} a_{p-2 i} a_{p-2 i-1} a_{p-2 i+1} \cdots a_{p} \in \mathcal{L}(P) .
$$

Note that $\left(w^{\prime}\right)^{\prime}=w$. Now exactly one of $w$ and $w^{\prime}$ has the descent $p-2 i-1$. The only other differences in the descent sets of $w$ and $w^{\prime}$ occur (possibly) for the numbers $p-2 i-2$ and $p-2 i$. Hence $(-1)^{\operatorname{comaj}(w)}+(-1)^{\operatorname{comaj}\left(w^{\prime}\right)}=0$. The surviving permutations $w=b_{1} \cdots b_{p}$ in $\mathcal{L}(P)$ are exactly those for which the chain of order ideals

$$
\emptyset \subset \cdots \subset\left\{b_{1}, b_{2}, \ldots, b_{p-4}\right\} \subset\left\{b_{1}, b_{2}, \ldots, b_{p-2}\right\} \subset\left\{b_{1}, b_{2}, \ldots, b_{p}\right\}=P
$$

is a $P$-domino tableau. We call $w$ a domino linear extension; they are in bijection with domino tableaux. Such permutations $w$ can only have descents in positions $p-j$ where $j$ is even, so $(-1)^{\operatorname{comaj}(w)}=1$. Hence (i) and (ii) are equal.

To prove that (ii) and (iii) are equal, let $\tau_{i}$ be the operator on $\mathcal{L}(P)$ defined by equation (2). Thus $w$ is self-evacuating if and only if

$$
w=w \tau_{1} \tau_{2} \cdots \tau_{p-1} \cdot \tau_{1} \cdots \tau_{p-2} \cdots \tau_{1} \tau_{2} \tau_{3} \cdot \tau_{1} \tau_{2} \cdot \tau_{1}
$$


On the other hand, note that $w$ is a domino linear extension if and only if

$$
w \tau_{p-1} \tau_{p-3} \tau_{p-5} \cdots \tau_{h}=w
$$

where $h=1$ if $p$ is even, and $h=2$ if $p$ is odd. It follows from Lemma 3.2 (letting $u=v=w$ ) that $w$ is a domino linear extension if and only if

$$
\widetilde{w}:=w \tau_{1} \cdot \tau_{3} \tau_{2} \tau_{1} \cdot \tau_{5} \tau_{4} \tau_{3} \tau_{2} \tau_{1} \cdots \tau_{m} \tau_{m-1} \cdots \tau_{1}
$$

is self-evacuating, where $m=p-1$ if $p$ is even, and $m=p-2$ if $p$ is odd. The proof follows since the map $w \mapsto \widetilde{w}$ is then a bijection between domino linear extensions and self-evacuating linear extensions of $P$.

The equivalence of (i) and (iii) above is an instance of Stembridge's " $q=-1$ phenomenon." Namely, suppose that an involution $\iota$ acts on a finite set $S$. Let $f: S \rightarrow \mathbb{Z}$. (Usually $f$ will be a "natural" combinatorial or algebraic statistic on $S$.) Then we say that the triple $(S, \iota, f)$ exhibits the $q=-1$ phenomenon if the number of fixed points of $\iota$ is given by $\sum_{t \in S}(-1)^{f(t)}$. See Stembridge [33][34][35]. The $q=-1$ phenomenon has been generalized to the action of cyclic groups by V. Reiner, D. Stanton, and D. White [23], where it is called the "cyclic sieving phenomenon." For further examples of the cyclic sieving phenomenon, see C. Bessis and V. Reiner [3], H. Barcelo, D. Stanton, and V. Reiner [1], and B. Rhoades [24]. In the next section we state a deep example of the cyclic sieving phenomenon, due to Rhoades, applied to the operator $\partial$ when $P$ is the product of two chains.

\section{Special cases.}

There are a few "nontrivial" classes of posets $P$ known for which the operation $\partial^{p}=\epsilon \epsilon^{*}$ can be described in a simple explicit way, so in particular the order of the dihedral group $D_{P}$ generated by $\epsilon$ and $\epsilon^{*}$ can be determined. There are also some "trivial" classes, such as hook shapes (a disjoint union of two chains with a $\hat{0}$ adjoined), where it is straightforward to compute the order of $\partial$ and $D_{P}$. The nontrivial classes of posets are all connected with the theory of standard Young tableaux or shifted tableaux, whose definition we assume is known to the reader. A standard Young tableau of shape $\lambda$ corresponds to a linear extension of a certain poset $P_{\lambda}$ in an obvious way, and similarly for a standard shifted tableau. (As mentioned in the introduction, Schützenberger originally defined evacuation for standard Young tableaux before extending it to linear extensions of any finite poset.) We will simply state the known results here. The posets will be defined by examples which should make the general definition clear. In these examples, the elements increase as we move down or to the right, so that the upper-left square is always the unique minimal element of $P_{\lambda}$.

Theorem 4.1. For the following shapes and shifted shapes $P$ with a total of $p=\# P$ squares, we have the indicated properties of $\partial^{p}$ and $D_{P}$. 


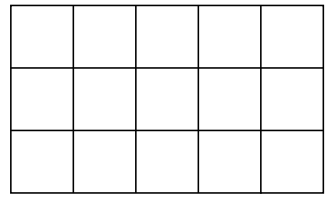

(a) rectangle

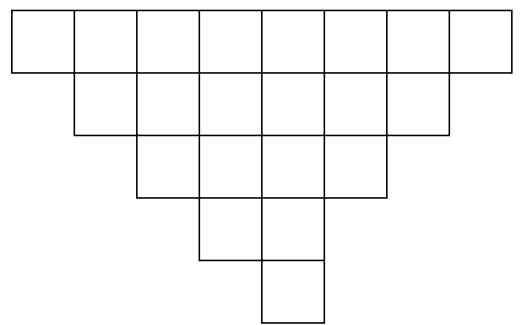

(c) shifted double staircase

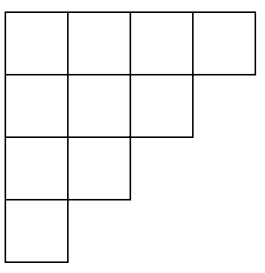

(b) staircase

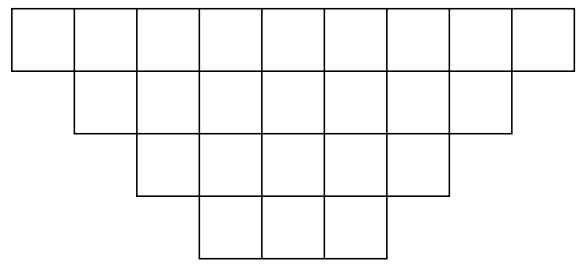

(d) shifted trapezoid

Figure 6: Some shapes and shifted shapes

(a) Rectangles (Figure 6(a)). Then $f \partial^{p}=f$ and $D_{P} \cong \mathbb{Z} / 2 \mathbb{Z}$ (if $m, n>1$ ). Moreover, if $f=\left(a_{i j}\right)$ (where we are regarding a linear extension of the rectangle $P$ as a labeling of the squares of $P)$, then $f \epsilon=\left(p+1-a_{m+1-i, n+1-j}\right)$.

(b) Staircases (Figure 6(b)). Then $f \partial^{p}=f^{t}$ (the transpose of $f$ ) and $D \cong \mathbb{Z} / 2 \mathbb{Z} \times \mathbb{Z} / 2 \mathbb{Z}$.

(c) Shifted double staircases (Figure $6(c)$ ). Then $f \partial^{p}=f$ and $D_{P} \cong \mathbb{Z} / 2 \mathbb{Z}$.

(d) Shifted trapezoids (Figure $6(d)$ ). Then $f \partial^{p}=f$ and $D_{P} \cong \mathbb{Z} / 2 \mathbb{Z}$.

Theorem 4.1(a) follows easily from basic properties of jeu de taquin due to Schützenberger [28] (see also [31, Ch. 7, Appendix 1]) and is often attributed to Schützenberger. We are unaware, however, of an explicit statement in the work of Schützenberger. Part (b) is due to Edelman and Greene [8, Cor. 7.23]. Parts (c) and (d) are due to Haiman [15, Thm. 4.4], who gives a unified approach also including (a) and (b).

The equivalence of (i) and (iii) in Theorem 3.1 was given a deep generalization by Rhoades [24] when $P$ is an $m \times n$ rectangular shape (so $p=m n$ ), as mentioned in the previous section. By Theorem 4.1(a) we have $f \partial^{p}=f$ when $P$ is a rectangular shape of size $p$. Thus every cycle of $\partial$, regarded as a permutation of the set $\mathcal{L}(P)$, has length $d$ dividing $p$. We can ask more generally for the precise cycle structure of $\partial$, i.e., the number of cycles of each length $d \mid p$. Equivalently, for any $d \in \mathbb{Z}$ (or just any $d \mid p$ ) we can ask for the quantity

$$
e_{d}(P)=\#\left\{f \in \mathcal{L}(P): f=f \partial^{d}\right\} .
$$


To answer this question, define the major index of the linear extension $f \in \mathcal{L}(P)$ by

$$
\operatorname{maj}(f)=\sum_{i} i
$$

where $i$ ranges over all entries of $P$ for which $i+1$ appears in a lower row than $i$ [31, p. 363]. For instance, if $f$ is given by

$$
f=\begin{array}{cccc}
1 & 3 & 4 & 8 \\
2 & 5 & 6 & 11 \\
7 & 9 & 10 & 12
\end{array}
$$

then $\operatorname{maj}(f)=1+4+6+8+11=30$. Let

$$
F(q)=\sum_{f \in \mathcal{L}(P)} q^{\operatorname{maj}(f)} .
$$

It is well known [31, Cor. 7.21.5] that

$$
F(q)=\frac{q^{n\left(\begin{array}{c}
m \\
2
\end{array}\right)}(1-q)\left(1-q^{2}\right) \cdots\left(1-q^{p}\right)}{\prod_{t \in P}\left(1-q^{h(t)}\right)},
$$

where $h(t)$ is the hook length of $t$. If say $m \leq n$, then we have more explicitly

$$
\begin{gathered}
\prod_{t \in P}\left(1-q^{h(t)}\right) \\
=[1][2]^{2}[3]^{3} \cdots[m]^{m}[m+1]^{m} \cdots[n]^{m}[n+1]^{m-1}[n+2]^{m-2} \cdots[n+m-1],
\end{gathered}
$$

where $[i]=1-q^{i}$. The beautiful result of Rhoades is the following.

Theorem 4.2. Let $P$ be an $m \times n$ rectangular shape. Set $p=m n$ and $\zeta=e^{2 \pi i / p}$. Then for any $d \in \mathbb{Z}$ we have

$$
e_{d}(P)=F\left(\zeta^{d}\right)
$$

Rhoades' proof of this theorem uses Kazhdan-Lusztig theory and a characterization of the dual canonical basis of $\mathbb{C}\left[x_{11}, \ldots, x_{n n}\right]$ due to Skandera [29]. Several questions are suggested by Theorems 4.1 and 4.2 .

1. Is there a more elementary proof of Theorem 4.2? For the special case of $2 \times n$ and $3 \times n$ rectangles, see [21]. The authors of [21] are currently hoping to extend their proof to general rectangles.

2. Can Theorem 4.2 be extended to more general posets, in particular, the posets of Theorem 4.1(b,c,d)?

3. Can Theorem 4.1 itself be extended to other classes of posets? A possible place to look is among the $d$-complete posets of Proctor [22]. Some work along these lines is being done by Kevin Dilks (in progress at the time of this writing). 


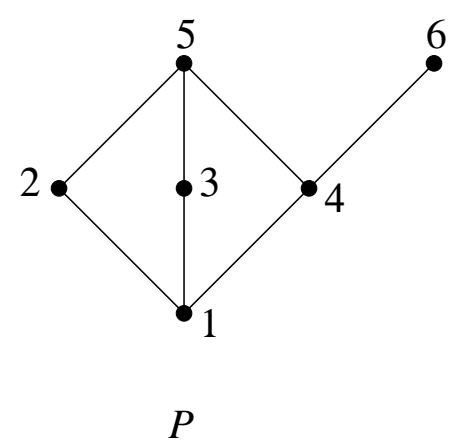

Figure 7: A linear extension of a poset $P$

\section{Growth diagrams}

There is an alternative approach to promotion and evacuation, kindly explained by an anonymous referee. This approach is based on the growth diagrams developed by S. Fomin in a series of papers [10][11][12][13]. In [31, pp. 424-429] Fomin uses growth diagrams to develop Schützenberger's work on evacuation related to the RSK algorithm. This approach can be extended to arbitrary posets by replacing Young diagrams with order ideals of $P$.

Let $f: P \rightarrow[p]$ be a linear extension of the $p$-element poset $P$. For simplicity we will denote the element $t \in P$ satisfying $f(t)=i$ by $i$. Figure 7 shows an example that we will use throughout this discussion.

We now define the growth diagram $\mathcal{D}(P, f)$ of the pair $(P, f)$. Begin with the points $(a, b) \in \mathbb{Z}^{2}$ satisfying $a, b \geq 0$ and $a+b \leq p$. We want to label each of these points $(a, b)$ with an order ideal $I(a, b)$ of $P$. In general we will have $\# I(a, b)=a+b$. We first label all the points satisfying $a+b=p$ with the elements $\{1,2, \ldots, p\}$ of the entire poset $P$, and the points $(0, b)$ with the order ideal $\{1,2, \ldots, b\}$. See Figure 8.

We now inductively label the remaining points according to the following local rule: suppose that we have labelled all the corners except the bottom-right corner of a unit square. The bottom-left corner $(a, b)$ will be labelled with an order ideal $I=I(a, b)$; the top-left corner $(a, b+1)$ will be labeled $I \cup\{i\}$ for some $1 \leq i \leq p$; and the top-right corner $(a+1, b+1)$ will be labelled $I \cup\{i, j\}$. We then define the labelling of the bottom-right corner $(a+1, b)$ by

$$
I(a+1, b)= \begin{cases}I(a, b) \cup\{i\}, & \text { if } i<j \text { in } P \\ I(a, b) \cup\{j\}, & \text { if } i \| j \text { in } P\end{cases}
$$

where $i \| j$ denotes that $i$ and $j$ are incomparable. The labelling begins at $(1, p-2)$ and works its way down and to the right. See Figure 9 for a diagram of the local rule and Figure 10 for the completed growth diagram of our example.

The bottom row of the growth diagram $\mathcal{D}(P, f)$ lists a chain $\emptyset=I_{0} \subset I_{1} \subset \cdots \subset$ $I_{p}=P$ of order ideals of $P$ with $\# I_{i}=i$. This chain corresponds to the linear extension $g$ of $P$ given by $g(t)=i$ if $t \in I_{i}-I_{i-1}$. Now every lattice path from $(0,0)$ to a point 


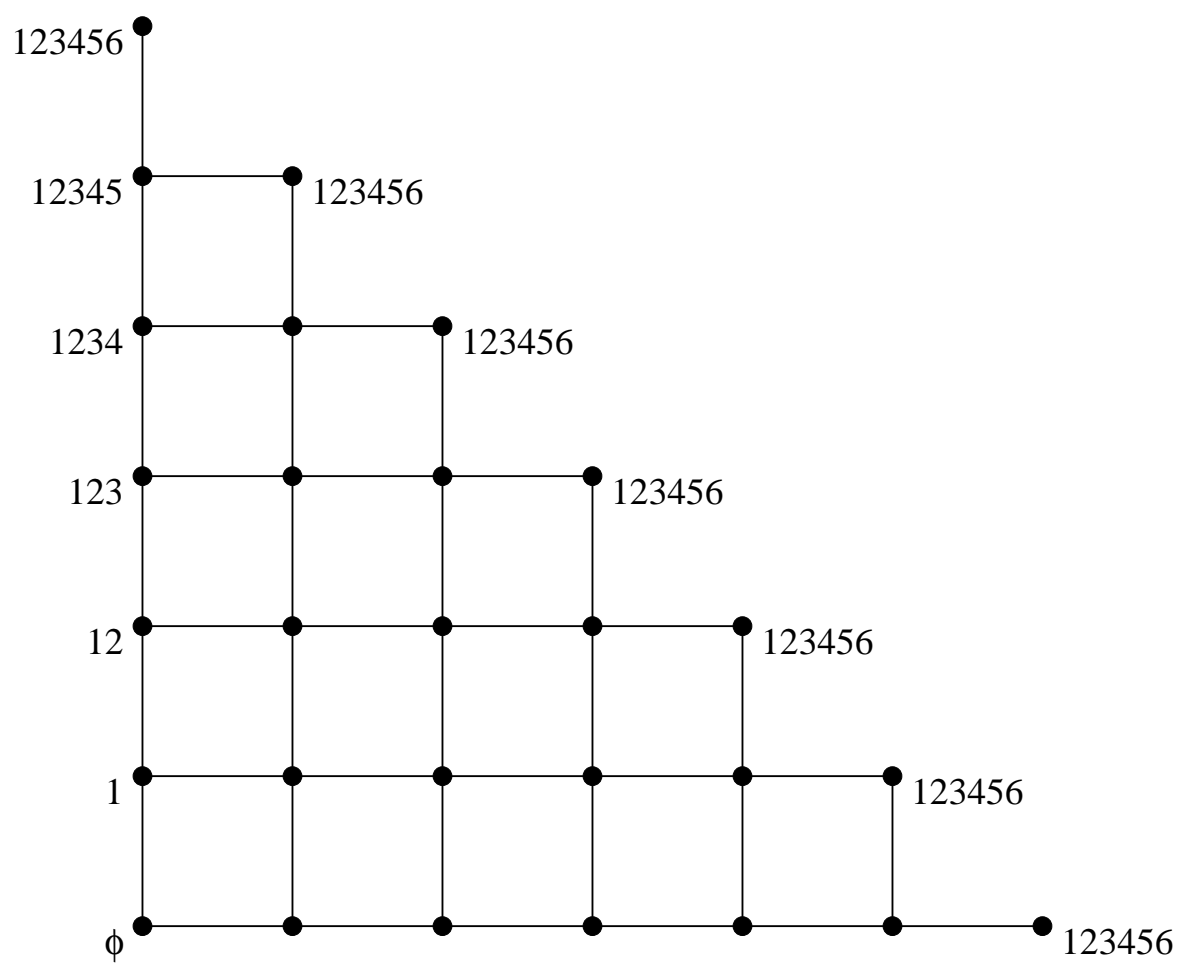

Figure 8: Initialization of the growth process

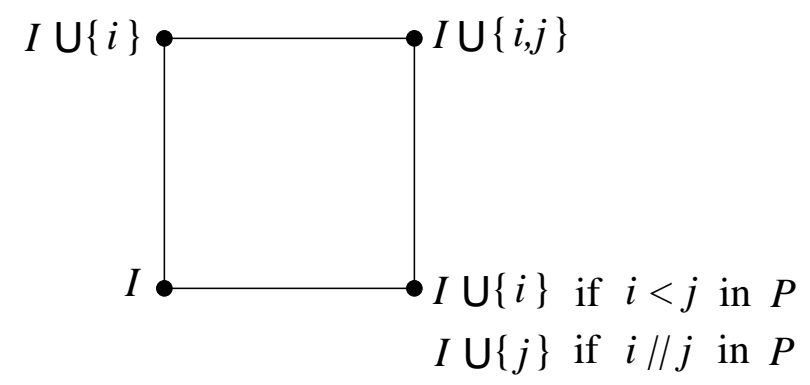

Figure 9: The local growth rule 


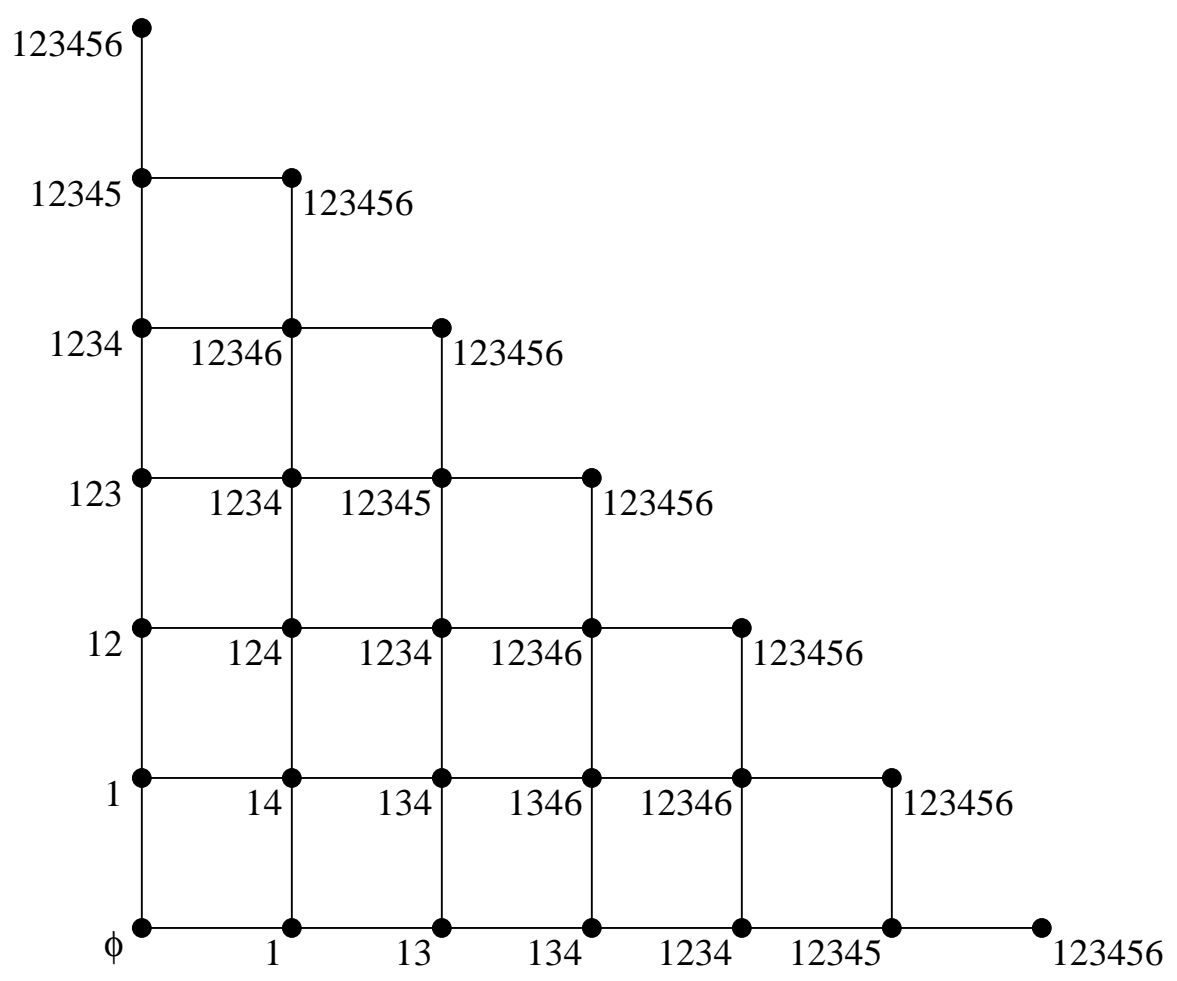

Figure 10: A growth diagram

(a.b) with $a+b=p$ with steps $(1,0)$ and $(0,1)$ defines a linear extension of $P$, just as we have done for the linear extension $g$. By analyzing how these linear extensions change as we alter the lattice path by changing two consecutive steps $(0,1),(1,0)$ to $(1,0),(0,1)$, we can deduce that $g=f \epsilon^{*}$, the dual evacuation of $f$. If we reflect $\mathcal{D}(P, f)$ about the main diagonal then we obtain $\mathcal{D}(P, g)=\mathcal{D}\left(P, \epsilon^{*}\right)$. Hence it is geometrically obvious that $\left(\epsilon^{*}\right)^{2}=1$. In a similar manner we can obtain the other parts of Theorem 2.1 and (with a little more work) Lemma 3.2.

\section{Generalizations.}

The basic properties of evacuation given in Sections 2 and 3 depend only on the formal properties of the group $G$ defined by equation (1). It is easy to find other examples of operators satisfying these conditions that are more general than the operators $\tau_{i}$ operating on linear extensions of posets. Hence the theory of promotion and evacuation extends to these more general situations.

Let $J(P)$ denote the set of all order ideals of the finite poset $P$, ordered by inclusion. By a well-known theorem of Birkhoff (see [30, Thm. 3.41]), the posets $J(P)$ coincide with the finite distributive lattices. There is a simple bijection [30, §3.5] between maximal chains $\emptyset=I_{0} \subset I_{1} \subset \cdots \subset I_{p}=P$ of $J(P)$ and linear extensions of $P$, viz., associate with this chain the linear extension $f: P \rightarrow[p]$ defined by $f(t)=i$ if $t \in I_{i}-I_{i-1}$. In terms 
of the maximal chain $\mathfrak{m}: \emptyset=I_{0} \subset I_{1} \subset \cdots \subset I_{p}=P$ of $J(P)$, the operator $\tau_{i}$ on linear extensions of $P$ can be defined as follows. The interval $\left[I_{i-1}, I_{i+1}\right]$ contains either three or four elements, i.e., either $I_{i}$ is the unique element satisfying $I_{i-1} \subset I_{i} \subset I_{i+1}$ or there is exactly one other such element $I^{\prime}$. In the former case define $\tau_{i}(\mathfrak{m})=\mathfrak{m}$; in the latter case, $\tau_{i}(\mathfrak{m})$ is obtained from $\mathfrak{m}$ by replacing $I_{i}$ with $I^{\prime}$.

The exact same definition of $\tau_{i}$ can be made for any finite graded poset, say for convenience with a unique minimal element $\hat{0}$ and unique maximal element $\hat{1}$, for which every interval of rank 2 contains either three or four elements. Let us call such posets slender. Clearly the $\tau_{i}$ 's satisfy the conditions (1). Thus Lemma 2.2 applies to the operators $\gamma, \gamma^{*}$, and $\delta$. (These observations seem first to have been made by van Leeuwen $[17, \S 2]$, after similar results by Malvenuto and Reutenauer [19] in the context of graphs rather than posets.) We also have an analogue for slender posets $Q$ of the equivalence of (ii) and (iii) in Lemma 3.2. The role of $P$-domino tableau is played by domino chains of $Q$, i.e., chains $\hat{0}=t_{0}<t_{1}<\cdots<t_{r}=\hat{1}$ in $P$ for which the interval $\left[t_{i-1}, t_{i}\right]$ is a two-element chain for $2 \leq i \leq r$, while $\left[t_{0}, t_{1}\right]$ is either a two-element or one-element chain (depending on whether the rank of $Q$ is even or odd). We then have that the number of self-evacuating maximal chains of $Q$ is equal to the number of domino chains of $Q$.

Some example of slender posets are Eulerian posets [30, §3.14], which include face posets of regular CW-spheres [4] and intervals in the Bruhat order of Coxeter groups $W$ (including the full Bruhat order of $W$ when $W$ is finite). Eulerian posets $Q$ have the property that every interval of rank 2 contains four elements. Hence there are no domino chains when $\operatorname{rank}(Q)>1$, and therefore also no self-evacuating maximal chains. NonEulerian slender posets include the weak order of a finite Coxeter group [5][6, Ch. 3] and face posets of regular CW-balls. We have not systematically investigated whether there are examples for which more can be said, e.g., an explicit description of evacuation or the determination of the order of the dihedral group generated by $\gamma$ and $\gamma^{*}$.

There is a simple example that can be made more explicit, namely, the face lattice $L_{n}$ of an $n$-dimensional cross-polytope $\mathcal{C}_{n}$ (the dual to an $n$-cube). The vertices of $\mathcal{C}_{n}$ can be labelled $1, \overline{1}, 2, \overline{2}, \ldots, n, \bar{n}$ so that vertices $i$ and $\bar{i}$ are antipodal for all $i$. A maximal chain $\hat{0}=t_{0}<t_{1}<\cdots<t_{n+1}=\hat{1}$ of $L_{n}$ can then be encoded as a signed permutation $a_{1} \cdots a_{n}$, i.e., take a permutation $b_{1} \cdots b_{n}$ and place bars above some subset of the $b_{i}$ 's. Thus $a_{i}$ is the unique vertex of the face $t_{i}$ that does not lie in $t_{i-1}$. Write' for the reversal of the bar, i.e., $i^{\prime}=\bar{i}$ and $\bar{i}^{\prime}=i$. Let $w=a_{1} \cdots a_{n}$ be a signed permutation of $1,2, \ldots, n$. Then it is easy to compute that

$$
\begin{aligned}
w \delta & =a_{2} a_{3} \cdots a_{n} a_{1}^{\prime} \\
w \gamma & =a_{1}^{\prime} a_{n} a_{n-1} \cdots a_{2} \\
w \gamma^{*} & =a_{n}^{\prime} a_{n-1}^{\prime} \cdots a_{1}^{\prime} \\
w \delta^{n+1}=w \gamma \gamma^{*} & =a_{2}^{\prime} a_{3}^{\prime} \ldots a_{n}^{\prime} a_{1} .
\end{aligned}
$$

Thus $\gamma \gamma^{*}$ has order $n$ if $n$ is odd and $2 n$ if $n$ is even. The dihedral group generated by $\gamma$ and $\gamma^{*}$ has order $2 n$ if $n$ is odd and $4 n$ if $n$ is even.

Can the concepts of promotion and evacuation be extended to posets that are not slender? We discuss one way to do this. Let $P$ be a graded poset of rank $n$ with $\hat{0}$ and $\hat{1}$. 
If $\mathfrak{m}: \hat{0}=t_{0}<t_{1}<\cdots<t_{n}=\hat{1}$ is a maximal chain of $P$, then we would like to define $\mathfrak{m} \tau_{i}$ so that (1) $\tau_{i}^{2}=1$, and (2) the action of $\tau_{i}$ is "local" at rank $i$, i.e., $\mathfrak{m} \tau_{i}$ should only involve maximal chains that agree with $\mathfrak{m}$ except possibly at $t_{i}$. There is no "natural" choice of a single chain $\mathfrak{m}^{\prime}=\mathfrak{m} \tau_{i}$, so we should be unbiased and choose a linear combination of chains. Thus let $K$ be a field of characteristic 0 . Write $\mathcal{M}(P)$ for the set of maximal chains of $P$ and $K \mathcal{M}(P)$ for the $K$-vector space with basis $\mathcal{M}(P)$. For $1 \leq i \leq n-1$ define a linear operator $\tau_{i}: K \mathcal{M}(P) \rightarrow K \mathcal{M}(P)$ as follows. Let $N_{i}(\mathfrak{m})$ be the set of maximal chains $\mathfrak{m}^{\prime}$ of $P$ that differ from $\mathfrak{m}$ exactly at $t_{i}$, i.e., $\mathfrak{m}^{\prime}$ has the form

$$
\mathfrak{m}^{\prime}: \hat{0}=t_{0}<t_{1}<\cdots<t_{i-1}<t_{i}^{\prime}<t_{i+1}<\cdots<t_{n}=\hat{1}
$$

where $t_{i}^{\prime} \neq t_{i}$. Suppose that $\# N_{i}(\mathfrak{m})=q \geq 1$. Then set

$$
\tau_{i}(\mathfrak{m})=\frac{1}{q+1}\left((q-1) \mathfrak{m}-2 \sum_{\mathfrak{m}^{\prime} \in N_{i}(\mathfrak{m})} \mathfrak{m}^{\prime}\right) .
$$

When $q=0$ we set $\mathfrak{m} \tau_{i}=\mathfrak{m}$, though it would make no difference to set $\mathfrak{m} \tau_{i}=-\mathfrak{m}$ to remain consistent with equation (6). It is easy to check that $\tau_{i}^{2}=1$. In fact, $\pm \tau_{i}$ are the unique involutions of the form $a \mathfrak{m}+b \sum_{\mathfrak{m}^{\prime} \in N_{i}(\mathfrak{m})} \mathfrak{m}^{\prime}$ for some $a, b \in K$ with $b \neq 0$ when $q \geq 1$. It is clear that also $\tau_{i} \tau_{j}=\tau_{j} \tau_{i}$ if $|j-i| \geq 2$, so the $\tau_{i}$ 's satisfy (1). Hence we can define promotion and evacuation on the maximal chains of any finite graded poset so that Lemma 2.2 holds, as well as an evident analogue of the equivalence of (ii) and (iii) in Theorem 3.1.

The obvious question then arises: are there interesting examples? We will discuss one example here, namely, the lattice $B_{n}(q)$ of subspaces of the $n$-dimensional vector space $\mathbb{F}_{q}^{n}$ (ordered by inclusion). This lattice is the " $q$-analogue" of the boolean algebra $B_{n}$ of all subsets of the set $\{1,2, \ldots, n\}$, ordered by inclusion. The boolean algebra $B_{n}$ is the lattice of order ideals of an $n$-element antichain $A$. Hence promotion and evacuation on the maximal chains of $B_{n}$ are equivalent to "classical" promotion and evacuation on $A$. The linear extensions of $A$ are just all the permutations $w$ of $\{1, \ldots, n\}$, and the evacuation $w \epsilon$ of $w=a_{1} a_{2} \cdots a_{n}$ is just the reversal $a_{n} \cdots a_{2} a_{1}$. Thus we are asking for a kind of $q$-analogue of reversing a permutation.

This problem can be reduced to a computation in the Hecke algebra $\mathcal{H}_{n}(q)$ of the symmetric group $\mathfrak{S}_{n}$ over the field $K$ (of characteristic 0). Recall (e.g., [16, §7.4]) that $\mathcal{H}_{n}(q)$ has generators $T_{1}, \ldots, T_{n-1}$ and relations

$$
\begin{aligned}
\left(T_{i}+1\right)\left(T_{i}-q\right) & =0 \\
T_{i} T_{j} & =T_{j} T_{i}, \quad|i-j| \geq 2 \\
T_{i} T_{i+1} T_{i} & =T_{i+1} T_{i} T_{i+1} .
\end{aligned}
$$

If $q=1$ then we have $T_{i}^{2}=1$, and the above relations are just the Coxeter relations for the group algebra $K \mathfrak{S}_{n}$.

For $1 \leq i \leq n-1$ let $s_{i}$ denote the adjacent transposition $(i, i+1) \in \mathfrak{S}_{n}$. A reduced decomposition of an element $w \in \mathfrak{S}_{n}$ is a sequence $\left(a_{1}, \ldots, a_{r}\right)$ of integers $1 \leq a_{i} \leq n-1$ 
such that $w=s_{a_{1}} \cdots s_{a_{r}}$ and $r$ is as small as possible, namely, $r$ is the number of inversions of $w$. Define $T_{w}=T_{a_{1}} \cdots T_{a_{r}}$. In particular, $T_{\mathrm{id}}=1$ and $T_{s_{k}}=T_{k}$. A standard fact about $\mathcal{H}_{n}(q)$ is that $T_{w}$ is independent of the choice of reduced decomposition of $w$, and the $T_{w}$ 's for $w \in \mathfrak{S}_{n}$ form a $K$-basis for $\mathcal{H}_{n}(q)$. We also have the multiplication rule

$$
T_{u} T_{k}= \begin{cases}T_{u s_{k}}, & \text { if } l\left(u s_{k}\right)=l(u)+1 \\ q T_{u s_{k}}+(q-1) T_{u}, & \text { if } l\left(u s_{k}\right)=l(u)-1\end{cases}
$$

for any $u \in \mathfrak{S}_{n}$.

Let $\operatorname{End}\left(K \mathcal{M}\left(B_{n}(q)\right)\right)$ be the set of all linear transformations

$$
K \mathcal{M}\left(B_{n}(q)\right) \rightarrow K \mathcal{M}\left(B_{n}(q)\right)
$$

Let

$$
t_{i}=-\frac{q+1}{2} \tau_{i}+\frac{q-1}{2} I
$$

the endomorphism sending a maximal chain $\mathfrak{m}$ to $\sum_{\mathfrak{m}^{\prime} \in N_{i}(\mathfrak{m})} \mathfrak{m}^{\prime}$. It is easy to check that the map $T_{i} \mapsto t_{i}$ extends to an algebra homomorphism (i.e., a representation of $\mathcal{H}_{n}(q)$ ) $\varphi: \mathcal{H}_{n}(q) \rightarrow \operatorname{End}\left(K \mathcal{M}\left(B_{n}(q)\right)\right)$. Moreover, $\varphi$ is injective. If we fix a maximal chain $\mathfrak{m}_{0}$, then the set $\mathcal{M}\left(B_{n}(q)\right)$ has a Bruhat decomposition $[14, \S 23.4]$

$$
\mathcal{M}\left(B_{n}(q)\right)=\bigsqcup_{w \in \mathfrak{S}_{n}} \Omega_{w}
$$

where $\bigsqcup$ denotes disjoint union and $\Omega_{\text {id }}=\left\{\mathfrak{m}_{0}\right\}$. Defining $t_{w}=\varphi\left(T_{w}\right)$, we then have

$$
t_{w}\left(\mathfrak{m}_{0}\right)=\sum_{\mathfrak{m} \in \Omega_{w}} \mathfrak{m}
$$

(In fact, this equation could be used to define $\Omega_{w}$.) Let $E_{i}=\frac{1}{q+1}\left(q-1-2 T_{i}\right) \in \mathcal{H}_{n}(q)$, so $E_{i}^{2}=1$. It follows that

$$
\mathfrak{m}_{0} \epsilon=\sum_{w \in \mathfrak{S}_{n}} c_{w}(q) \sum_{\mathfrak{m} \in \Omega_{w}} w
$$

where $c_{w}(q)$ is defined by the Hecke algebra expansion

$$
E_{1} E_{2} \cdots E_{n-1} E_{1} E_{2} \cdots E_{n-2} \cdots E_{1} E_{2} E_{1}=\sum_{w \in \mathfrak{S}_{n}} c_{w}(q) T_{w}
$$

Note that by Lemma 2.2(a) the right-hand side of equation (8) remains invariant if we reverse the order of the factors on the left-hand side. In general, however, the expression $E_{a_{1}} \cdots E_{a_{r}}$ is not the same for all reduced decompositions $\left(a_{1}, \ldots, a_{r}\right)\left(r=\left(\begin{array}{l}n \\ 2\end{array}\right)\right)$ of $w_{0}=$ $n, n-1, \ldots, 1$. 
When $w \in \mathfrak{S}_{4}$ the values of $c_{w}(q)$ are given by

$$
\begin{aligned}
& c_{1234}(q)=(q-1)^{2} /(q+1)^{2} \\
& c_{1243}(q)=-2(q-1)^{3} /(q+1)^{4} \\
& c_{1324}(q)=-16 q(q-1)\left(q^{2}+1\right) /(q+1)^{6} \\
& c_{1342}(q)=4(q-1)^{2} /(q+1)^{4} \\
& c_{1423}(q)=4(q-1)^{2} /(q+1)^{4} \\
& c_{1432}(q)=-8(q-1)^{3} /(q+1)^{6} \\
& c_{2134}(q)=-2(q-1)^{3} /(q+1)^{4} \\
& c_{2143}(q)=4(q-1)^{2} /(q+1)^{4} \\
& c_{2314}(q)=-4(q-1)^{4} /(q+1)^{6} \\
& c_{2341}(q)=-8(q-1) /(q+1)^{4} \\
& c_{2413}(q)=0 \\
& c_{2431}(q)=16(q-1)^{2} /(q+1)^{6} \\
& c_{3124}(q)=-4(q-1)^{4} /(q+1)^{6} \\
& c_{3142}(q)=0 \\
& c_{3214}(q)=8(q-1)^{3} /(q+1)^{6} \\
& c_{3241}(q)=0 \\
& c_{3412}(q)=16(q-1)^{2} /(q+1)^{6} \\
& c_{3421}(q)=-32(q-1) /(q+1)^{6} \\
& c_{4123}(q)=-8(q-1) /(q+1)^{4} \\
& c_{4132}(q)=16(q-1)^{2} /(q+1)^{6} \\
& c_{4213}(q)=0 \\
& c_{4231}(q)=-32(q-1) /(q+1)^{6} \\
& c_{4312}(q)=-32(q-1) /(q+1)^{6} \\
& c_{4321}(q)=64 /(q+1)^{6} . \\
& =0
\end{aligned}
$$

Although many values of $c_{w}(q)$ appear to be "nice," not all are as nice as the above data suggests. For instance,

$$
\begin{gathered}
c_{12453}(q)=4\left(q^{2}+6 q+1\right)(q-1)^{4} /(q+1)^{8} \\
c_{13245}(q)=-2\left(q^{4}-8 q^{3}-2 q^{2}-8 q+1\right)(q-1)^{5} /(q+1)^{10} \\
c_{13425}(q)=-4\left(q^{6}-6 q^{5}-33 q^{4}+12 q^{3}-33 q^{2}-6 q+1\right)(q-1)^{2} / \\
\quad(q+1)^{10} .
\end{gathered}
$$

We will prove two results about the $c_{w}(q)$ 's.

Theorem 6.1. Let id denote the identity permutation in $\mathfrak{S}_{n}$. Then

$$
c_{\mathrm{id}}(q)=\left(\frac{q-1}{q+1}\right)^{\lfloor n / 2\rfloor} .
$$


Proof (sketch). I am grateful to Monica Vazirani for assistance with the following proof. Define a scalar product on $\mathcal{H}_{n}(q)$ by

$$
\left\langle T_{u}, T_{v}\right\rangle=q^{\ell(u)} \delta_{u v}
$$

where $\ell(u)$ denotes the number of inversions of $u$ (i.e., the length of $u$ as an element of the Coxeter group $\left.\mathfrak{S}_{n}\right)$. Then one can check that for any $g, h \in \mathcal{H}_{n}(q)$ we have

$$
\left\langle T_{i} g, h\right\rangle=\left\langle g, T_{i} h\right\rangle
$$

and

$$
\left\langle g T_{i}, h\right\rangle=\left\langle g, h T_{i}\right\rangle
$$

Since $E_{i}^{2}=1$ it follows that

$$
\left\langle E_{i} g E_{i}, 1\right\rangle=\langle g, 1\rangle
$$

Now

$$
c_{\mathrm{id}}(q)=\left\langle E_{1} E_{2} \cdots E_{n-1} E_{1} E_{2} \cdots E_{n-2} \cdots E_{1} E_{2} E_{1}, 1\right\rangle .
$$

Using equation (9) and the commutation relation $E_{i} E_{j}=E_{j} E_{i}$ if $|i-j| \geq 2$, we obtain

$$
c_{\mathrm{id}}(q)=\left\langle E_{n-1} E_{n-3} \cdots E_{r}, 1\right\rangle,
$$

where $r=1$ if $n$ is even, and $r=2$ if $n$ is odd. For any subset $S$ of $\{n-1, n-3, \ldots, r\}$ we have

$$
\prod_{i \in S} T_{i}=T_{\prod_{i \in S} s_{i}} .
$$

(The $T_{i}$ 's and $s_{i}$ 's for $i \in S$ commute, so the above products are well-defined.) Hence we obtain the scalar product $\left\langle E_{n-1} E_{n-3} \cdots E_{r}, 1\right\rangle$ by setting $T_{i}=0$ in each factor of the product $E_{n-1} E_{n-2} \cdots E_{r}$, so we get

$$
\left\langle E_{n-1} E_{n-3} \cdots E_{r}, 1\right\rangle=\left(\frac{q-1}{q+1}\right)^{\lfloor n / 2\rfloor},
$$

completing the proof.

If $w=a_{1} a_{2} \cdots a_{n} \in \mathfrak{S}_{n}$, then write $\widehat{w}$ for the reversal $a_{n} \cdots a_{2} a_{1}$. Equivalently, $\widehat{w}=w_{0} w$, where $w_{0}=n, n-1, \ldots, 1$ (the longest permutation in $\mathfrak{S}_{n}$ ). Our second result on the polynomials $c_{w}(q)$ is the following.

Theorem 6.2. Let $w \in \mathfrak{S}_{n}$, and let $\kappa(w)$ denote the number of cycles of $w$. Then $c_{w}(q)$, regarded as a rational function of $q$, has numerator divisible by $(q-1)^{n-\kappa(\widehat{w})}$.

Proof. Consider the coefficient of $T_{w}$ in the expansion of the product on the left-hand side of (8). For each factor $E_{i}=\frac{1}{q+1}\left(q-1-2 T_{i}\right)$ we must choose a term $(q-1) /(q+1)$ or $-2 T_{i} /(q+1)$. If we choose $(q-1) /(q+1)$ then we have introduced a factor of $q-1$. If we choose $-2 T_{i} /(q+1)$ and multiply some $T_{u}$ by it, then a $T_{v}$ so obtained satisfies either $v=u s_{i}$ or $v=u$; in the latter case a factor of $q-1$ is introduced. It follows that every 
contribution to the coefficient of $T_{w}$ arises from choosing a subsequence $\left(b_{1}, \ldots, b_{j}\right)$ of the reduced decomposition $(1,2, \ldots, p-1,1,2, \ldots, p-2, \ldots, 1,2,1)$ of $w_{0}$ such that

$$
w=s_{b_{1}} \cdots s_{b_{j}}
$$

in which case we will obtain a factor $(q-1)^{\left(\begin{array}{c}n \\ 2\end{array}\right)-j}$. The $b_{i}$ 's correspond to the terms that do not introduce a factor of $q-1$.

Now let $\boldsymbol{a}=\left(a_{1}, \ldots, a_{\left(\begin{array}{c}n \\ 2\end{array}\right)}\right)$ be a reduced decomposition of $w_{0}$. It is a well-known and simple consequence of the strong exchange property for reduced decompositions (e.g. [6, Thm. 1.4.3]) that if $k$ is the length of the longest subsequence $\left(b_{1}, \ldots, b_{k}\right)$ of $\boldsymbol{a}$ such that $s_{b_{1}} \cdots s_{b_{k}}=w$, then $\left(\begin{array}{l}n \\ 2\end{array}\right)-k$ is the minimum number of transpositions $t_{1}, \ldots, t_{k}$ for which $w=w_{0} t_{1} \cdots t_{k}$. This number is just $n-\kappa\left(w_{0}^{-1} w\right)=n-\kappa\left(w_{0} w\right)=n-\kappa(\widehat{w})$, so $k=\left(\begin{array}{l}n \\ 2\end{array}\right)-n+\kappa(\widehat{w})$.

It follows that the largest possible value of $j$ in equation $(10)$ is $\left(\begin{array}{l}n \\ 2\end{array}\right)-n+\kappa(\widehat{w})$. Thus $\left(\begin{array}{l}n \\ 2\end{array}\right)-j \geq n-\kappa(\widehat{w})$, completing the proof.

Theorem 6.2 need not be best possible. For instance, some values of $c_{w}(1)$ can be 0 , such as $c_{2413}(q)$. For a nonzero example, we have that $(q-1)^{4}$ divides $c_{2314}(q)$, but $4-\kappa(4132)=2$.

\section{References}

[1] H. Barcelo, D. Stanton, and V. Reiner, Bimahonian distributions, arXiv:math/0703479.

[2] A. Berenstein and A. N. Kirillov, Domino tableaux, Schützenberger involution, and the symmetric group action, Discrete Math. 225 (2000), 15-24.

[3] C. Bessis and V. Reiner, Cyclic sieving of noncrossing partitions for complex reflection groups, arXiv:math/0701792.

[4] A. Björner, Posets, regular CW complexes and Bruhat order, European J. Combin. 5 (1984), 7-16.

[5] A. Björner, Orderings of Coxeter groups, in Combinatorics and Algebra, Boulder 1983 (C. Greene, ed.), Contemp. Math., vol. 34, American Mathematical Society, Providence, RI, 1984, pp. 175-195.

[6] A. Björner and F. Brenti, Combinatorics of Coxeter Groups, Graduate Texts in Mathematics 231, Springer-Verlag, New York, 2005.

[7] P. Cameron and D. G. Fon-Der-Flaass, Orbits of antichains revisited, European J. Combin. 16 (1995), 545-554.

[8] P. H. Edelman and C. Greene, Balanced tableaux, Advances in Math. 63 (1987), 42-99.

[9] P. Edelman, T. Hibi, and R. Stanley, A recurrence for linear extensions, Order 6 (1989), 15-18. 
[10] S. Fomin, Two-dimensional growth in Dedekind lattices, M. S. thesis, Leningrad State University, 1979.

[11] S. Fomin, Generalized Robinson-Schensted-Knuth correspondence, Zapiski Nauchn. Sem. LOMI 155 (1986), 156-175 (in Russian).

[12] S. Fomin, Duality of graded graphs, J. Algebraic Combinatorics 3 (1994), 357-404.

[13] S. Fomin, Schensted algorithms for dual graded graphs, J. Algebraic Combinatorics 4 (1995), 5-45.

[14] W. Fulton and J. Harris, Representation Theory, Graduate Texts in Mathematics 129, Springer-Verlag, New York, 1991.

[15] M. D. Haiman, Dual equivalence with applications, including a conjecture of Proctor, Discrete Math. 99 (1992), 79-113.

[16] J. E. Humphreys, Reflection Groups and Coxeter Groups, Cambridge Studies in Advanced Mathematics 29, Cambridge University Press, Cambridge, 1990.

[17] M. A. A. van Leeuwen, The Robinson-Schensted and Schützenberger algorithms, an elementary approach, The Foata Festschrift, Electron. J. Combin. 3 (1996), R15.

[18] M. A. A. van Leeuwen, Flag varieties and interpretations of Young tableau algorithms, J. Algebra 224 (2000), 397-426.

[19] C. Malvenuto and C. Reutenauer, Evacuation of labelled graphs, Discrete Math. 132 (1994), 137-143.

[20] D. I. Panyushev, On orbits of antichains of positive roots, European J. Combin. 30 (2009), 586-594.

[21] T. K. Petersen, P. Pylyavskyy, and B. Rhoades, Promotion and cyclic sieving via webs, preprint, arXiv:0904.3375.

[22] R. A. Proctor, Overview of recent research concerning the hook length and jeu de taquin properties and $d$-complete posets, http://www . math . unc . edu/Faculty/rap/RROvrvw. html.

[23] V. Reiner, D. Stanton, and D. White, The cyclic sieving phenomenon, J. Combinatorial Theory Ser. A 108 (2004), 17-50.

[24] B. Rhoades, Cyclic sieving, promotion, and representation theory, Ph.D. thesis, University of Minnesota, 2008.

[25] M.-P. Schützenberger, Quelques remarques sur une construction de Schensted, Canad. J. Math. 13 (1961), 117-128.

[26] M.-P. Schützenberger, Promotion des morphismes d'ensembles ordonnés, Discrete Math. 2 (1972), 73-94.

[27] M.-P. Schützenberger, Evacuations, in Colloquio Internazionale sulle Teorie Combinatorie (Rome, 1973), Tomo I, Atti dei Convegni Lincei, No. 17, Accad. Naz. Lincei, Rome, 1976, pp. 257-264.

[28] M.-P. Schützenberger, La correspondance de Robinson, in Combinatoire et représentation du groupe symétrique (Actes Table Ronde CNRS, Univ. Louis-Pasteur 
Strasbourg, Strasbourg, 1976), Lecture Notes in Math., Vol. 579, Springer, Berlin, 1977, pp. 59-113.

[29] M. Skandera, On the dual canonical and Kazhdan-Lusztig bases and 3412, 4231avoiding permutations, preprint, www.lehigh.edu/ mas906/papers/tnncone.pdf.

[30] R. Stanley, Enumerative Combinatorics, vol. 1, Wadsworth and Brooks/Cole, Pacific Grove, CA, 1986; second printing, Cambridge University Press, New York/Cambridge, 1996.

[31] R. Stanley, Enumerative Combinatorics, vol. 2, Cambridge University Press, New York/Cambridge, 1999.

[32] R. Stanley, Some remarks on sign-balanced and maj-balanced poset, Advances in Applied Math. 34 (2005), 880-902.

[33] J. R. Stembridge, Some hidden relations involving the ten symmetry classes of plane partitions, J. Combinatorial Theory Ser. A 68 (1994), 372-409.

[34] J. R. Stembridge, On minuscule representations, plane partitions and involutions in complex Lie groups, Duke Math. J. 73 (1994), 469-490.

[35] J. R. Stembridge, Canonical bases and self-evacuating tableaux, Duke Math. J. 82 (1996), 585-606.

[36] G. P. Tesler, Semi-primary lattices and tableau algorithms, Ph.D. thesis, M.I.T., 1995. 